\title{
Study of Dual to Ratio-Cum-Product Estimator of Finite Population Mean under Double Sampling in Sample Surveys
}

\author{
Sanjib Choudhury* \\ Department of Mathematics \\ National Institute of Technology Nagaland \\ Nagaland, India-797103 \\ Email: sanjibchy07@gmail.com \\ Bhupendra Kumar Singh \\ Department of Mathematics \\ North Eastern Regional Institute of Science and Technology \\ Arunachal Pradesh, India-791109 \\ E-mail: bksinghnerist@gmail.com \\ Received 10 October 2011 \\ Accepted 7 July 2014
}

\begin{abstract}
A class of estimator based on dual to ratio and dual to product-type estimator in double sampling for estimating finite population mean is proposed. The bias and mean square error of the proposed estimator are obtained in two different cases. A comparative study has been made with usual estimators, dual to ratio estimator, dual to product type estimator in double sampling in two different cases. The proposed estimator is found to be an improvement over the other estimators as special cases.

Keywords: Dual to Ratio-Cum- Product Estimator, Double Sampling, Finite Population Mean, Mean Square Error, Efficiency.
\end{abstract}

AMS 2000 Subject Classifications: 62D05.

${ }^{*}$ Corresponding author 


\section{Introduction}

In sample surveys, auxiliary information is used at either selection or estimation stage or both to improve the precision of the estimate of the population parameter. The literature on survey sampling describes several methods of using the auxiliary variable at the estimation stage. This includes among others; linear regression estimator, ratio estimator, product estimator and difference estimator. When the correlation between study variate and auxiliary variate is positive (high), the ratio method of estimation is most preferable for estimating the population mean. On the other hand, if this correlation is negative, product method of estimation is used. Cochran (1940) used auxiliary information at estimation stage and proposed ratio estimator. Robson (1957) and Murthy (1964) envisaged product estimator, Searls (1964) used coefficient of variation of study variate, motivated by Searls (1964), Sisodia and Dwivedi (1981) utilized coefficient of variation of auxiliary variate. Singh (1969) proposed ratio-cum-product estimator, Srivenkataramana (1980), first proposed dual to ratio estimator, Bandyopadhyay (1980) introduced dual variables for estimation of population parameters, Singh et al. (2005) proposed dual to ratio-cum-product estimator, to suggest modified estimators in order to provide better alternatives for estimation of finite population mean.

When the population mean $\bar{X}$ of the auxiliary variable $X$ is unknown before start of the survey, it is sometimes estimated from a preliminary large sample on which only the auxiliary characteristic $x$ is observed. The value of $\bar{X}$ in the estimator is then replaced by its estimate. A smaller second-phase sample of the study variate $y$ is then taken. This technique is known as double sampling or two-phase sampling. Neyman (1938) was the first to give the concept of double sampling in connection with collecting information on the strata sizes in a stratified sampling. For more applications of double sampling method, the reader is referred to Sukhatme \& Koshal (1959), Singh and Singh (1965), Singh (1968), Chand (1975), Mukerjee et al. (1987), Dorfman (1994), Rao \& Sitter (1995), Prasad et al. (1996), Breslow \& Hilubkov (1997), Singh \& Espejo (2000), Singh \& Espejo (2007), Bhushan et al. (2008), Singh et al. (2010).

Let $U=\left(u_{1}, u_{2}, \ldots, u_{N}\right)$ be a finite population of size $\mathrm{N}$ units, $y$ and $(x, z)$ are the study and auxiliary variates respectively. When the population mean $\bar{X}($ or $\bar{Z})$ of $x($ or $z)$ is not known, a first-phase sample of size $n_{1}$ is drawn from the population on which only $x($ or $z)$ characteristics is measured in order to furnish a good estimate of $\bar{X}($ or $\bar{Z})$. After then a second-phase sample of size $n$ such that $n<n_{1}$ is drawn on which both the variates $y$ and $x($ or $z)$ are measured.

Thus, the usual ratio and product estimators in double sampling are respectively as

$$
\bar{y}_{R}^{d}=\bar{y} \frac{\bar{x}_{1}}{\bar{x}} \text { and } \bar{y}_{P}^{d}=\bar{y} \frac{\bar{z}}{\bar{z}_{1}},
$$

where $\bar{x}, \bar{y}$ and $\bar{z}$ are the sample mean of $x, y$ and $Z$ respectively based on the sample size $n$ out of the population $N$ units and $\bar{x}_{1}=\frac{1}{n_{1}} \sum_{i=1}^{n_{1}} x_{i} \quad$ and $\bar{Z}_{1}=\frac{1}{n_{1}} \sum_{i=1}^{n_{1}} z_{i}$ denote the sample mean of $X$ and $Z$ respectively based on the first-phase sample of the size $n_{1}$.

Singh (1969) improved the ratio and product methods of estimation and suggested the 'ratio-cumproduct' estimator for $\bar{Y}$ as

$$
\bar{Y}_{R P}=\bar{y} \frac{\bar{X}}{\bar{x}} \frac{\bar{z}}{\bar{Z}}
$$

where $\bar{X}=\frac{1}{N} \sum_{i=1}^{N} x_{i}$ and $\bar{Z}=\frac{1}{N} \sum_{i=1}^{N} z_{i}$.

Considering the transformations

$x_{i}^{*}=\left(1+g^{\prime}\right) \bar{X}-g^{\prime} x_{i}$ and $z_{i}^{*}=\left(1+g^{\prime}\right) \bar{Z}-g^{\prime} z_{i}$, where $i=1,2,3, \ldots, N$ and $g^{\prime}=n /(N-n)$, Singh et al. (2005) suggested dual to ratio-cum-product estimator as

$$
\bar{y}_{R P}^{*}=\bar{y} \frac{\bar{x}^{*}}{\bar{X}} \frac{\bar{Z}}{\bar{Z}^{*}}
$$

where $\bar{X}^{*}=\left(1+g^{\prime}\right) \bar{X}-g \bar{X}$ and $\bar{Z}^{*}=\left(1+g^{\prime}\right) \bar{Z}-g^{\prime} \bar{Z}$ are unbiased estimators for $\bar{X}$ and $\bar{Z}$ respectively and $\operatorname{corr}\left(\bar{y}, \bar{x}^{*}\right)=-\rho_{y x}$ and $\operatorname{corr}\left(\bar{y}, \bar{z}^{*}\right)=-\rho_{y z}$.

Using the transformation $x_{i}^{* d}=(1+g) \bar{x}_{1}-g x_{i}$, $i=1,2,3 \ldots N$, where $g=n /\left(n_{1}-n\right)$, Kumar et al. 
(2006) obtained dual to ratio estimator in double sampling is given as

$$
\bar{y}_{R}^{* d}=\bar{y} \frac{\bar{x}^{* d}}{\bar{x}_{1}}
$$

where $\bar{X}^{* d}=(1+g) \bar{X}_{1}-g \bar{X}$ is an unbiased estimator for $\bar{X}$ and the correlation of $\left(\bar{y}, \bar{x}^{* d}\right)$ is negative.

Let $z_{i}^{* d}=(1+g) \bar{z}_{1}-g z_{i}, \quad$ then clearly $\bar{Z}^{* d}=(1+g) \bar{Z}_{1}-g \bar{Z}$ is also unbiased estimator for $\bar{Z}$ and the correlation of $\left(\bar{y}, \bar{z}^{* d}\right)$ is negative. Utilizing this transformation, Singh and Choudhury (2012) have considered dual to product estimator in double sampling as

$$
\bar{y}_{P}^{* d}=\bar{y} \frac{\bar{z}_{1}}{\bar{z}^{* d}} \text {. }
$$

Motivated by Singh et al. (2005), we have studied the properties of 'ratio-cum-product' estimator suggested by Singh (1969) using the transformations $x_{i}^{* d}$ and $z_{i}^{* d}$ in double sampling.

\section{The Adopted Class of Estimator}

The proposed dual to ratio-cum-product estimator of population mean $\bar{Y}$ in double sampling is given as

$$
\bar{y}_{R P}^{* d}=\bar{y} \frac{\bar{x}^{* d}}{\bar{x}_{1}} \frac{\bar{z}_{1}}{\bar{z}^{* d}}
$$

To obtain the bias (B) and mean square error (MSE) of $\bar{y}_{R P}^{* d}$, we write

$e_{0}=(\bar{y}-\bar{Y}) / \bar{Y}, e_{1}=(\bar{x}-\bar{X}) / \bar{X}$,

$e_{1}^{\prime}=\left(\bar{X}_{1}-\bar{X}\right) / \bar{X}, e_{2}=(\bar{Z}-\bar{Z}) / \bar{Z}$ and

$e_{2}^{\prime}=\left(\bar{Z}_{1}-\bar{Z}\right) / \bar{Z}$.

such that

$$
E\left(e_{0}\right)=E\left(e_{1}\right)=E\left(e_{1}^{\prime}\right)=E\left(e_{2}\right)=E\left(e_{2}^{\prime}\right)=0
$$

The following notations are used hereafter

$C_{y}=\frac{S_{y}}{\bar{Y}}, \quad C_{x}=\frac{S_{x}}{\bar{X}}$ and $C_{z}=\frac{S_{z}}{\bar{Z}}$ are the coefficient of variations of the study variate $y$, auxiliary variates $X$ and $Z$ respectively.

$\rho_{y x}=\frac{S_{y x}}{S_{x} S_{y}}, \quad \rho_{y z}=\frac{S_{y z}}{S_{y} S_{z}}$ and $\rho_{z x}=\frac{S_{z x}}{S_{z} S_{x}}$ are the correlation coefficients between $y$ and $x, y$ and $z$ and
$X$ and $Z$ respectively.

$S_{y}^{2}=\frac{1}{N-1} \sum_{i=1}^{N}\left(y_{i}-\bar{Y}\right)^{2}, \quad S_{x}^{2}=\frac{1}{N-1} \sum_{i=1}^{N}\left(x_{i}-\bar{X}\right)^{2}$

and $S_{z}^{2}=\frac{1}{N-1} \sum_{i=1}^{N}\left(z_{i}-\bar{Z}\right)^{2}$ are the population variances of study variate $y$, auxiliary variates $X$ and $Z$ respectively.

$S_{y x}=\frac{1}{N-1} \sum_{i=1}^{N}\left(y_{i}-\bar{Y}\right)\left(x_{i}-\bar{X}\right)$

$S_{y z}=\frac{1}{N-1} \sum_{i=1}^{N}\left(y_{i}-\bar{Y}\right)\left(z_{i}-\bar{Z}\right)$

and

$S_{x z}=\frac{1}{N-1} \sum_{i=1}^{N}\left(x_{i}-\bar{X}\right)\left(z_{i}-\bar{Z}\right) \quad$ are the co-variances between $y$ and $x, y$ and $z$; and $x$ and $z$ respectively. Expressing $\bar{y}_{R P}^{* d}$ in terms of $e$ 's, we obtain

$$
\begin{aligned}
& \bar{y}_{R P}^{* d}=\bar{Y}\left(1+e_{0}+e_{2}^{\prime}+e_{0} e_{2}^{\prime}\right)\left\{1+(1+g) e_{1}^{\prime}-g e_{1}\right\} \\
& \times\left\{1+e_{1}^{\prime}+(1+g)\left(e_{2}^{\prime}+e_{1}^{\prime} e_{2}^{\prime}\right)-g\left(e_{2}+e_{1}^{\prime} e_{2}\right)\right\}^{-1}
\end{aligned}
$$

Assume that $\left|e_{1}^{\prime}+(1+g)\left(e_{2}^{\prime}+e_{1}^{\prime} e_{2}^{\prime}\right)-g\left(e_{2}+e_{1}^{\prime} e_{2}\right)\right|<1$ and the sample size is large enough so that $\left\{1+e_{1}^{\prime}+(1+g)\left(e_{2}^{\prime}+e_{1}^{\prime} e_{2}^{\prime}\right)-g\left(e_{2}+e_{1}^{\prime} e_{2}\right)\right\}^{-1}$ is expandable.

Expanding the right hand side of eq. (2) and neglecting terms of $e$ 's of power greater than two, we have

$$
\begin{aligned}
& \bar{y}_{R P}^{* d}=\bar{Y}\left\{1+e_{0}+g\left(-e_{1}+e_{1}^{\prime}+e_{2}-e_{2}^{\prime}-e_{1}^{\prime 2}-e_{0} e_{1}+e_{0} e_{1}^{\prime}\right.\right. \\
& \left.+e_{0} e_{2}-e_{0} e_{2}^{\prime}+e_{1} e_{1}^{\prime}\right)+g^{2}\left(-e_{1} e_{2}+e_{1} e_{2}^{\prime}+e_{1}^{\prime} e_{2}-e_{1}^{\prime} e_{2}^{\prime}+e_{2}^{2}\right) \\
& \left.-(1+2 g) e_{2} e_{2}^{\prime}+g(1+g) e_{2}^{\prime 2}+\ldots .\right\}
\end{aligned}
$$

or

$$
\begin{aligned}
& \bar{y}_{R P}^{* d}-\bar{Y} \cong \bar{Y}\left\{e_{0}+g\left(-e_{1}+e_{1}^{\prime}+e_{2}-e_{2}^{\prime}-e_{1}^{\prime 2}-e_{0} e_{1}+e_{0} e_{1}^{\prime}\right.\right. \\
& \left.+e_{0} e_{2}-e_{0} e_{2}^{\prime}+e_{1} e_{1}^{\prime}\right)+g^{2}\left(-e_{1} e_{2}+e_{1} e_{2}^{\prime}+e_{1}^{\prime} e_{2}-e_{1}^{\prime} e_{2}^{\prime}+e_{2}^{2}\right) \\
& \left.-(1+2 g) e_{2} e_{2}^{\prime}+g(1+g) e_{2}^{\prime 2}\right\}
\end{aligned}
$$

For the bias and MSE, the following two cases are considered separately. 
Case I: When the second-phase sample of size $n$ is a subsample of the first phase of size $n_{1}$.

Case II: When the second phase sample of size $n$ is drawn independently of the first phase sample of size $n_{1}$.

\section{Bias, Mean Square Error of $\bar{y}_{R P}^{* d}$ in Case $\mathbf{I}$}

In Case I, we have

$E\left(e_{0}\right)=E\left(e_{1}\right)=E\left(e_{1}^{\prime}\right)=E\left(e_{2}\right)=E\left(e_{2}^{\prime}\right)=0, E\left(e_{0}^{2}\right)=\frac{1-f}{n} C_{y}^{2}$,

$E\left(e_{1}^{2}\right)=\frac{1-f}{n} C_{x}^{2}, E\left(e_{1}^{\prime 2}\right)=\frac{1-f_{1}}{n_{1}} C_{x}^{2}, E\left(e_{2}^{2}\right)=\frac{1-f}{n} C_{z}^{2}$,

$E\left(e_{2}^{\prime 2}\right)=\frac{1-f_{1}}{n_{1}} C_{z}^{2}, E\left(e_{0} e_{1}\right)=\frac{1-f}{n} k_{y x} C_{x}^{2}, E\left(e_{0} e_{1}^{\prime}\right)=\frac{1-f_{1}}{n_{1}} k_{y x} C_{x}^{2}$,

$E\left(e_{0} e_{2}\right)=\frac{1-f}{n} k_{y z} C_{z}^{2}, E\left(e_{0} e_{2}^{\prime}\right)=\frac{1-f_{1}}{n_{1}} k_{y z} C_{z}^{2}, E\left(e_{1} e_{1}^{\prime}\right)=\frac{1-f_{1}}{n_{1}} C_{x}^{2}$,

$E\left(e_{1} e_{2}\right)=\frac{1-f}{n} k_{z x} C_{x}^{2}, E\left(e_{1} e_{2}^{\prime}\right)=\frac{1-f_{1}}{n_{1}} k_{z x} C_{x}^{2}, E\left(e_{1}^{\prime} e_{2}\right)=\frac{1-f_{1}}{n_{1}} k_{z x} C_{x}^{2}$,

$E\left(e_{1}^{\prime} e_{2}^{\prime}\right)=\frac{1-f_{1}}{n_{1}} k_{z x} C_{x}^{2}, \quad E\left(e_{2} e_{2}^{\prime}\right)=\frac{1-f_{1}}{n_{1}} C_{z}^{2}$,

where $f=\frac{n}{N}, \quad f_{1}=\frac{n_{1}}{N}, k_{y x}=\rho_{y x} \frac{C_{y}}{C_{x}}$,

$k_{y z}=\rho_{y z} \frac{C_{y}}{C_{z}}$ and $k_{z x}=\rho_{x z} \frac{C_{z}}{C_{x}}$.

Taking expectation in eq. (3) and using the results given in (4), we get the bias of the estimator $\bar{y}_{R P}^{* d}$ to the first degree of approximation as

$B\left(\bar{y}_{R P}^{* d}\right)_{I}=\bar{Y} \frac{1-f^{*}}{n} g\left(-k_{y x} C_{x}^{2}+k_{y z} C_{z}^{2}+g C_{z}^{2}\right)$,

where $f^{*}=n / n_{1}$. The bias, $B\left(\bar{y}_{R P}^{* d}\right)_{I}$ in eq. (5) is 'zero' if $g=\left(k_{y x} C_{x}^{2}-k_{y z} C_{z}^{2}\right) / C_{z}^{2}$.

Thus, the estimator $\bar{y}_{R P}^{* d}$ with $g=\left(k_{y x} C_{x}^{2}-k_{y z} C_{z}^{2}\right) / C_{z}^{2}$ is almost unbiased.

To obtain the MSE of the proposed class of estimator, eq. (3) can be written as

$$
\bar{y}_{R P}^{* d}-\bar{Y} \cong \bar{Y}\left\{e_{0}+g\left(-e_{1}+e_{1}^{\prime}+e_{2}-e_{2}^{\prime}\right)\right\}
$$

Squaring both sides in eq. (6), taking expectation and using the results given in (4), we obtain the MSE of the proposed estimator $\bar{y}_{R P}^{* d}$ to the first degree of approximation is given as

$$
\begin{aligned}
\operatorname{MSE}\left(\bar{y}_{R P}^{* d}\right)_{I}= & \bar{Y}^{2}\left[\frac{1-f}{n} C_{y}^{2}+\frac{1-f^{*}}{n} g\left\{2\left(k_{y z} C_{z}^{2}-k_{y x} C_{x}^{2}\right)\right.\right. \\
& \left.\left.+g\left(C_{x}^{2}+C_{z}^{2}-2 k_{z x} C_{x}^{2}\right)\right\}\right]
\end{aligned}
$$

\subsection{Remark}

If the information of the auxiliary variates $Z$ and $Z_{1}$ are not used, i.e., if $Z$ and $Z_{1}$ are considered to be a nonzero constants, the estimator $\bar{y}_{R P}^{* d}$ in eq. (1) reduces to dual to ratio estimator $\bar{y}_{R}^{* d}$ in double sampling suggested by Kumar et al. (2006). The bias and MSE of $\bar{y}_{R}^{* d}$ can be obtained by omitting the terms $\kappa_{y z}, \kappa_{z x}$ and $C_{z}^{2}$ in the eq.'s (5) and (7) respectively, as

$$
B\left(\bar{y}_{R}^{* d}\right)_{I}=-\bar{Y} \frac{1-f^{*}}{n} g k_{y x} C_{x}^{2}
$$

and

$$
\operatorname{MSE}\left(\bar{y}_{R}^{* d}\right)_{I}=\bar{Y}^{2}\left\{\frac{1-f}{n} C_{y}^{2}+\frac{1-f^{*}}{n} C_{x}^{2} g\left(g-2 k_{y x}\right)\right\} .
$$

\subsection{Remark}

If the information of the auxiliary variates $X$ and $X_{1}$ are not used, i.e., if $X$ and $X_{1}$ are considered to be a nonzero constants, the estimator $\bar{y}_{R P}^{* d}$ in eq. (1) reduces to dual to product estimator $\bar{y}_{P}^{* d}$ in double sampling suggested by Singh and Choudhury (2012). The bias and MSE of $\bar{y}_{P}^{* d}$ can be obtained by omitting the terms $\kappa_{y x}, \kappa_{z x}$ and $C_{x}^{2}$ in the eq.'s (5) and (7) respectively as

$$
B\left(\bar{y}_{P}^{* d}\right)_{I}=\bar{Y} \frac{1-f^{*}}{n} g C_{z}^{2}\left(k_{y z}+g\right)
$$

and

$$
\operatorname{MSE}\left(\bar{y}_{P}^{* d}\right)_{I}=\bar{Y}^{2}\left\{\frac{1-f}{n} C_{y}^{2}+\frac{1-f^{*}}{n} g C_{z}^{2}\left(g+2 k_{y z}\right)\right\} .
$$




\section{Efficiency Comparisons of $\bar{y}_{R P}^{* d}$ in Case I}

In this section, firstly, we compare MSE of conventional estimators $\bar{y}, \bar{y}_{R}^{d}$ and $\bar{y}_{P}^{d}$ with MSE of proposed estimator $\bar{y}_{R P}^{* d}$.

The MSE of sample mean $\bar{y}$ under SRSWOR sampling scheme is given by

$$
M(\bar{y})=\frac{1-f}{n} \bar{Y}^{2} C_{y}^{2}
$$

From eq.'s (7) and (10), it is observed that the proposed estimator $\bar{y}_{R P}^{* d}$ is better than $\bar{y}$ if

$$
\frac{k_{y x} C_{x}^{2}-k_{y z} C_{z}^{2}}{C_{x}^{2}+C_{Z}^{2}-2 k_{z x} C_{x}^{2}}>\frac{1}{2} g .
$$

To compare the usual ratio estimator $\bar{y}_{R}^{d}$ in double sampling, the MSE of $\bar{y}_{R}^{d}$ to the first degree of approximation is given as

$$
\operatorname{MSE}\left(\bar{y}_{R}^{d}\right)_{I}=\bar{Y}^{2}\left\{\frac{1-f}{n} C_{y}^{2}+\frac{1-f^{*}}{n} C_{x}^{2}\left(1-2 k_{y x}\right)\right\}
$$

From eq.'s (7) and (12) that the estimator $\bar{y}_{R P}^{* d}$ has smaller MSE than the usual ratio estimator $\bar{y}_{R}^{d}$ in double sampling if

$$
C_{x}^{2}(1-g)\left(1+g-2 k_{y x}\right)>g C_{z}^{2}\left\{g+2\left(k_{y z}-g k_{x z}\right)\right\},
$$

where $k_{x z}=\frac{\rho_{z x} C_{x}}{C_{z}}$.

This condition holds if

$$
\begin{aligned}
& n_{1}>2 n, k_{y z}<(1+g) / 2 \text { and } \\
& g<-2 k_{y z} /\left(1-2 k_{x z}\right)
\end{aligned}
$$

Further, we compare MSE of the proposed estimator $\bar{y}_{R P}^{* d}$ with usual product estimator $\bar{y}_{P}^{d}$ in double sampling. The MSE of $\bar{y}_{P}^{d}$ to the first degree of approximation is given as

$$
\operatorname{MSE}\left(\bar{y}_{P}^{d}\right)_{I}=\bar{Y}^{2}\left\{\frac{1-f}{n} C_{y}^{2}+\frac{1-f^{*}}{n} C_{z}^{2}\left(1+2 k_{y z}\right)\right\}
$$

From eq.'s (7) and (14), it is found that the proposed estimator $\bar{y}_{R P}^{* d}$ will dominate over the usual product estimator $\bar{y}_{P}^{d}$ in double sampling if $C_{z}^{2}(1-g)\left(1+g+2 k_{y z}\right)>C_{x}^{2} g\left\{g\left(1-2 k_{z x}\right)-2 k_{y x}\right\}$.

This condition holds if

$$
n_{1}>2 n, \frac{1+g}{2}>-k_{y z} \text { and } g<\frac{k_{y x}}{0.5-k_{x x}}
$$

Secondly, comparing the MSE between the proposed estimator $\bar{y}_{R P}^{* d}$ and dual to ratio estimator $\bar{y}_{R}^{* d}$ in double sampling.

We note from eq.'s (7) and (8) that the estimator $\bar{y}_{R P}^{* d}$ has smaller MSE than dual to ratio estimator $\bar{y}_{R}^{* d}$ in double sampling if

$$
\frac{2 k_{y z} C_{z}^{2}}{2 k_{z x} C_{x}^{2}-C_{z}^{2}}<g .
$$

Lastly, we compare the MSE of the proposed estimator $\bar{y}_{R P}^{* d}$ with dual to product estimator $\bar{y}_{P}^{* d}$ in double sampling suggested by Singh and Choudhury (2012).

From eq.'s (7) and (9), it is found that the proposed estimator $\bar{y}_{R P}^{* d}$ is better than $\bar{y}_{P}^{* d}$ if

$$
\frac{2 k_{y x}}{1-2 k_{z x}}>g
$$

Therefore, it seems from the above results that the proposed estimator $\bar{y}_{R P}^{* d}$ may be made better than other estimators under stated conditions.

\section{Bias and MSE of $\bar{y}_{R P}^{* d}$ in Case II}

In Case II, we have 


$$
\left.\begin{array}{l}
E\left(e_{0}\right)=E\left(e_{1}\right)=E\left(e_{1}^{\prime}\right)=E\left(e_{2}\right)=E\left(e_{2}^{\prime}\right)=0, E\left(e_{0}^{2}\right)=\frac{1-f}{n} C_{y}^{2}, \\
E\left(e_{1}^{2}\right)=\frac{1-f}{n} C_{x}^{2}, E\left(e_{1}^{2}\right)=\frac{1-f_{1}}{n_{1}} C_{x}^{2}, E\left(e_{2}^{2}\right)=\frac{1-f}{n} C_{z}^{2}, \\
E\left(e_{2}^{\prime 2}\right)=\frac{1-f_{1}}{n_{1}} C_{z}^{2}, E\left(e_{0} e_{1}\right)=\frac{1-f}{n} k_{y x} C_{x}^{2}, E\left(e_{0} e_{1}^{\prime}\right)=0, \\
E\left(e_{0} e_{2}\right)=\frac{1-f}{n} k_{y z} C_{z}^{2}, E\left(e_{0} e_{2}^{\prime}\right)=0, E\left(e_{1} e_{1}^{\prime}\right)=0, E\left(e_{1} e_{2}\right)=\frac{1-f}{n} k_{x x} C_{x}^{2}, \\
E\left(e_{1} e_{2}^{\prime}\right)=0, E\left(e_{1}^{\prime} e_{2}\right)=0, E\left(e_{1}^{\prime} e_{2}^{\prime}\right)=\frac{1-f_{1}}{n_{1}} k_{x x} C_{x}^{2}, E\left(e_{2} e_{2}^{\prime}\right)=0 .
\end{array}\right\}
$$

Taking expectation in eq. (3) and using the results given in (18), we get the bias of $\bar{y}_{R P}^{* d}$ to the first degree of approximation as

$$
\begin{aligned}
B\left(\bar{y}_{R P}^{* d}\right)_{I I} & =\bar{Y} g\left[\frac{1-f}{n}\left\{\left(k_{y z} C_{z}^{2}-k_{y x} C_{x}^{2}\right)+g\left(k_{z x} C_{x}^{2}+C_{z}^{2}\right)\right\}\right. \\
+ & \left.\frac{1-f_{1}}{n_{1}}\left\{\left(C_{z}^{2}-C_{x}^{2}\right)+g\left(C_{z}^{2}-k_{z x} C_{x}^{2}\right)\right\}\right]
\end{aligned}
$$

The bias, $B\left(\bar{y}_{R P}^{* d}\right)_{I I}$ in eq. (19) is 'zero' if

$$
g=\frac{\frac{1-f}{n}\left(k_{y x} C_{x}^{2}-k_{y z} C_{z}^{2}\right)+\frac{1-f_{1}}{n_{1}}\left(C_{x}^{2}-C_{z}^{2}\right)}{\frac{1-f}{n}\left(k_{z x} C_{x}^{2}+C_{z}^{2}\right)+\frac{1-f_{1}}{n_{1}}\left(C_{z}^{2}-k_{z x} C_{x}^{2}\right)}
$$

Thus, the proposed estimator $\bar{y}_{R P}^{* d}$ with the value of ' $g$ ' is almost unbiased.

Squaring both sides in eq. (6), then taking expectation and using the results given in (18), we obtain the MSE of the estimator $\bar{y}_{R P}^{* d}$ to the first degree of approximation as

$$
\begin{gathered}
\operatorname{MSE}\left(\bar{y}_{R P}^{* d}\right)_{I I}=\bar{Y}^{2}\left[\frac{1-f}{n}\left\{C_{y}^{2}+2 g\left(k_{y z} C_{z}^{2}-k_{y x} C_{x}^{2}\right)\right\}\right. \\
\left.+\mu g^{2}\left(C_{x}^{2}+C_{z}^{2}-2 k_{z x} C_{x}^{2}\right)\right]
\end{gathered}
$$

where $\mu=\frac{1-f}{n}+\frac{1-f_{1}}{n_{1}}$.

\subsection{Remark}

If the information of the auxiliary variates $Z$ and $Z_{1}$ are not used, i.e., if $Z$ and $Z_{1}$ are considered to be a non- zero constants, the estimator $\bar{y}_{R P}^{* d}$ in eq. (1) reduces to dual to ratio estimator $\bar{y}_{R}^{* d}$ in double sampling. The bias and MSE of $\bar{y}_{R}^{* d}$ can be obtained by omitting the terms $\kappa_{y z}, \kappa_{z x}$ and $C_{z}^{2}$ in the eq.'s (19) and (20) respectively as

$$
B\left(\bar{y}_{R}^{* d}\right)_{I I}=-\bar{Y} g C_{x}^{2}\left(\frac{1-f_{1}}{n_{1}}+\frac{1-f}{n} k_{y x}\right)
$$

and

$$
\operatorname{MSE}\left(\bar{y}_{R}^{* d}\right)_{I I}=\bar{Y}^{2}\left\{\frac{1-f}{n} C_{y}^{2}-g C_{x}^{2}\left(2 \frac{1-f}{n} k_{y x}-\mu g\right)\right\} .
$$

\subsection{Remark}

If the information of the auxiliary variates $X$ and $X_{1}$ are not used, i.e., if $x$ and $x_{1}$ are considered to be a nonzero constants, the estimator $\bar{y}_{R P}^{* d}$ in eq. (1) reduces to dual to product estimator $\bar{y}_{P}^{* d}$ in double sampling. The bias and MSE of $\bar{y}_{P}^{* d}$ can be obtained by omitting the terms $\kappa_{y x}, \kappa_{z x}$ and $C_{x}^{2}$ in the eq.'s (19) and (20) respectively as

$$
B\left(\bar{y}_{P}^{* d}\right)_{I I}=\bar{Y} g C_{z}^{2}\left(\frac{1-f}{n} k_{y z}+\frac{1-f_{1}}{n_{1}}+\mu g\right)
$$

and

$$
\operatorname{MSE}\left(\bar{y}_{P}^{* d}\right)_{I I}=\bar{Y}^{2}\left\{\frac{1-f}{n} C_{y}^{2}+g C_{z}^{2}\left(2 \frac{1-f}{n} k_{y z}+\mu g\right)\right\}
$$

\section{Efficiency Comparisons of $\bar{y}_{R P}^{* d}$ in Case II}

Firstly, we compare the MSE of conventional estimators $\bar{y}, \bar{y}_{R}^{d}$ and $\bar{y}_{P}^{d}$ with the MSE of proposed estimator $\bar{y}_{R P}^{* d}$.

From eq.'s (10) and (20), it is observed that the proposed estimator $\bar{y}_{R P}^{* d}$ is better than $\bar{y}$ if

$$
\frac{B}{A}<-\frac{1}{2} g
$$

where $A=\mu\left(C_{x}^{2}+C_{z}^{2}-2 k_{z x} C_{x}^{2}\right)$ and 


$$
B=\frac{1-f}{n}\left(k_{y z} C_{z}^{2}-k_{y x} C_{x}^{2}\right)
$$

To compare with the usual ratio estimator $\bar{y}_{R}^{d}$ in double sampling, the MSE of $\bar{y}_{R}^{d}$ to the first degree of approximation as

$$
\operatorname{MSE}\left(\bar{y}_{R}^{d}\right)_{I I}=\bar{Y}^{2}\left(\frac{1-f}{n} C_{y}^{2}-2 \frac{1-f}{n} k_{y x} C_{x}^{2}+\mu C_{x}^{2}\right)
$$

We note from eq.'s (20) and (24) that the estimator $\bar{y}_{R P}^{* d}$ has smaller MSE than usual ratio estimator $\bar{y}_{R}^{d}$ if

$$
\left.\begin{array}{l}
\text { either } \frac{-B-\sqrt{B^{2}+A C}}{A}<g<\frac{-B+\sqrt{B^{2}+A C}}{A} \\
\text { or } \quad \frac{-B+\sqrt{B^{2}+A C}}{A}<g<\frac{-B-\sqrt{B^{2}+A C}}{A}
\end{array}\right\}
$$

where $C=\mu C_{x}^{2}-2 \frac{1-f}{n} k_{y x} C_{x}^{2}$.

Further, we compare MSE of the proposed estimator $\bar{y}_{R P}^{* d}$ with usual product estimator $\bar{y}_{P}^{d}$ in double sampling. To the first degree of approximation, the MSE of $\bar{y}_{P}^{d}$ is given as

$$
\operatorname{MSE}\left(\bar{y}_{P}^{d}\right)_{I I}=\bar{Y}^{2}\left(\frac{1-f}{n} C_{y}^{2}+2 \frac{1-f}{n} k_{y z} C_{z}^{2}+\mu C_{z}^{2}\right)
$$

From eq.'s (20) and (26), it is found that the proposed estimator $\bar{y}_{R P}^{* d}$ will dominate over the usual product estimator $\bar{y}_{P}^{d}$ in double sampling if

$$
\left.\begin{array}{l}
\text { either } \frac{-B-\sqrt{B^{2}+A C^{\prime}}}{A}<g<\frac{-B+\sqrt{B^{2}+A C^{\prime}}}{A} \\
\text { or } \quad \frac{-B+\sqrt{B^{2}+A C^{\prime}}}{A}<g<\frac{-B-\sqrt{B^{2}+A C^{\prime}}}{A}
\end{array}\right\}
$$

where $C^{\prime}=\mu C_{z}^{2}+2 \frac{1-f}{n} k_{y z} C_{z}^{2}$.

Secondly, comparing the MSE between the proposed estimator $\bar{y}_{R P}^{* d}$ and dual to ratio estimator $\bar{y}_{R}^{* d}$ in double sampling.
We note from eq.'s (20) and (21) that the estimator $\bar{y}_{R P}^{* d}$ has smaller MSE than dual to ratio estimator $\bar{y}_{R}^{{ }^{d} d}$ in double sampling if

$$
g>\frac{2 \frac{1-f}{n} k_{y z} C_{z}^{2}}{\mu\left(2 k_{z x} C_{x}^{2}-C_{z}^{2}\right)} .
$$

Lastly, we compare the MSE of the proposed estimator $\bar{y}_{R P}^{* d}$ with dual to product estimator $\bar{y}_{P}^{* d}$ in double sampling.

From eq.'s (20) and (22), it is observed that the proposed estimator $\bar{y}_{R P}^{* d}$ is better than $\bar{y}_{P}^{* d}$ if

$$
g>-\frac{2 \frac{1-f}{n} k_{y x}}{\mu\left(2 k_{z x}-1\right)} \text {. }
$$

\section{Conclusion}

We have developed an efficient class of estimator based on ratio-cum-product estimator, suggested by Singh (1969) and unbiased transformation of Kumar et al. (2006). The comparative study shows that the proposed estimator $\bar{y}_{R P}^{* d}$ established their superiority over sample mean $\bar{y}$, ratio estimator $\bar{y}_{R}^{d}$ and product estimator $\bar{y}_{P}^{d}$ in double sampling if the condition referred to eq.'s (11), (13) and (15) for Case I; and eq.'s (23), (25) and (27) for Case II are satisfied.

Also, the comparison with Kumar et al. (2006) estimator of dual to ratio and Singh and Choudhury (2012) estimator of dual to product-type in double sampling shows that the proposed estimator is better, provided the conditions $\left\{2 k_{y z} C_{z}^{2} /\left(2 k_{z x} C_{x}^{2}-C_{z}^{2}\right)\right\}<g$ referred in eq. (16) and $\left\{2 k_{y x} /\left(1-2 k_{z x}\right)\right\}>g$ referred in eq. (17) for Case $\mathrm{I}$; and $g>\left\{2 \frac{1-f}{n} k_{y z} C_{z}^{2} / \mu\left(2 k_{z x} C_{x}^{2}-C_{z}^{2}\right)\right\}$ referred to eq. (28), $g>\left\{-2 \frac{1-f}{n} k_{y x} / \mu\left(2 k_{z x}-1\right)\right\}$ refer to eq. (29) for Case II are satisfied respectively.

\section{References}

1. A. H. Dorfman, A note on variance estimation for the regression estimator in double sampling, Journal of the American Statistical Association 89(1994) 137-140. 
2. B. K. Singh and S. Choudhury, Dual to Product Estimator for Estimating Population Mean in Double Sampling, International Journal of Statistics and Systems 7(1) (2012) 31-39.

3. B. Prasad, R. S. Singh and H. P. Singh, Some chain ratiotype estimators for ratio of two population means using two auxiliary characters in two phase sampling, Metron 54(1996) 95-113.

4. B. V. S. Sisodia and V. K. Dwivedi, A modified ratio estimator using coefficient of variation of auxiliary variable. Jour. Ind. Soc. Agril. Statist. 33(1981) 13-18.

5. B. V. Sukhatme and R. S. Koshal, A contribution to double sampling. Journal of the Indian Society of Agricultural Statistics 11(1959) 128-144.

6. D. S. Robson, Applications of multivariate polykays to the theory of unbiased ratio-type estimation, Journal of the American Statistical Association 52(1957) 511-522.

7. D. Singh, Double sampling and its application in agriculture, in: Contributions in Statistics and Agricultural Sciences, 213-226, (New Delhi: Indian Society of Agricultural Statistics, 1968).

8. D. Singh and B. D. Singh Double sampling for stratification on successive occasions, Journal of the American Statistical Association 60(1965) 784-792.

9. D. T. Searls, The utilization of known coefficient of variation in the estimation procedure, Journal of American Statistical Association 59(1964) 1125-26.

10. H. P. Singh and M. R. Espejo, An improved class of chain regression estimators in two phase sampling. Statistics \& Decisions 18(2000) 205-218.

11. H. P. Singh and M. R. Espejo, Double sampling ratioproduct estimator of a finite population mean in sampling surveys. Journal of Applied Statistics 34(1) (2007) 71-85.

12. H. P. Singh and S. Kumar, Improved estimation of population mean under two-phase sampling with subsampling the non-respondents. Journal of Statistical Planning and Inference 140(9) (2010) 2536-2550.

13. H. P. Singh, R. Singh, M. R. Espejo, M. D. Pineda and S. Nadarajan, On the efficiency of the dual to ratio-cumproduct estimator. Mathematical Proceedings of the Royal Irish Academy 105 A (2) (2005) 51-56.

14. J. N. K. Rao and R. R. Sitter, Variance estimation under two phase sampling with application to imputation for missing data, Biometrika 82(1995) 453-460.

15. J. Neyman, Contribution to the theory of sampling human populations, Journal of the American Statistical Association 33(1938) 101-116.

16. L. Chand, Some ratio-type estimators based on two or more auxiliary variables. PhD Thesis, (Ames, Iowa State University, 1975).

17. M. Kumar and S. Bhal, Class of dual to ratio estimators for double sampling, Statistics Papers 47(2006), 319326.

18. M. P. Singh, Comparison of some ratio-cum-product estimators. Sankhya Series B 31(1969) 375-382.

19. M. N. Murthy, Product method of estimation, Sankhya A 26(1964) 69-74.
20. N. E. Breslow and R. Holubkov, Maximum likelihood estimation of logistic regression parameters under twophase outcome-dependent sampling, Journal of the Royal Statistical Society (Series B) 59(1997) 447-461.

21. R. Mukerjee, T. J. Rao, and K. Vijayan, Regression type estimator using multiple auxiliary information, Australian Journal of Statistics 29(1987) 244-254.

22. S. Bandyopadhyay, Improved ratio and product estimators, Sankhya Series C 42(2) (1980) 45-49.

23. S. Bhushan, A. Pandey and S. Katara, A class of estimators in double sampling using two auxiliary variables, Journal of Reliability and Statistical Studies 1(2008), 67-73.

24. T. Srivenkataramana, A dual to ratio estimator in sample surveys. Biometrika 67(1) (1980) 199-204.

25. W. G. Cochran, Sampling Techniques, (Third U. S. Edition. Wiley Eastern Limited, 1977). 